methylmalonic aciduria, and 21-hydroxylase deficiency in one 3 month old and one previously diagnosed 5 year old. Four patients were previously healthy, and suffered fever, seizures, coma, acidosis, and hypoglycemia soon after vaccination, all succumbing to respiratory failure before 2 years of age. Symmetric foci, cystic cavitations with neuronal loss, and vascular proliferation were found at autopsy. (Yang Y, Sujan S, Sun F et al. Acute metabolic crisis induced by vaccination in seven Chinese patients. Pediatr Neurol 2006;35:114-118). (Respond: Dr Yang, Department of Pediatrics, Peking University First Hospital, Beijing, 100034, China).

COMMENT. One patient had a family history of infantile deaths and was diagnosed with congenital adrenal hyperplasia and 21-hydroxylase deficiency before vaccination. A second patient subsequently diagnosed with $21-\mathrm{OH}$ deficiency was malnourished in infancy, and a 3 month-old with methylmalonic aciduria was hypotonic. The remaining 4 infants appeared normal before vaccination. Vaccination in infants with unrecognized metabolic disorders carries a risk of metabolic crisis. Infants who are malnourished, slow in development, hypotonic or have symptoms suggesting a metabolic disorder should receive appropriate laboratory tests before vaccination.

\title{
NEURAL CORRELATES OF AUTISM
}

\section{GAIT DISORDERS IN AUTISM}

Gait in 11 children with autism (age range $4-7$ years) and 11 controls was analyzed, using the GAITRite electronic walkway connected to a computer in a study at Department of Psychological Medicine, Monash University, Clayton, Victoria, Australia. Children with autism had difficulty walking tandem, reduced stride regularity, and variable velocity, compatible with cerebellar dysfunction. They were also less coordinated and erratic in their movements. Postural abnormalities in head and trunk suggested basal ganglia involvement. Abnormal gait is proposed as a useful clinical screening test for autism. (Rinehart NJ, Tonge $\mathrm{BJ}$, Iansek R et al. Gait function in newly diagnosed children with autism: cerebellar and basal ganglia related motor disorder. Dev Med Child Neurol Oct 2006;48:819-824). (Respond: Dr Rinehart, Department of Psychological Medicine, Monash University, Level 3, Block P, Monash Medical Centre, Clayton, Victoria 3168, Australia).

COMMENT. Neuropathological findings in autism (Palmen SJMC et al. Brain 2004;127:2572-2583; Ped Neur Briefs Dec 2004;18:89-90) document an increase in head circumference, brain weight and brain volume, decrease in Purkinje cells in the cerebellum, and dysgenesis in the cerebral cortex. Autism is a neurodevelopmental, genetically determined disorder characterized by impairments in social interaction and communication skills, cognitive rigidity, abnormal language development, and repetitive, stereotypical behaviors.

A significant increase in prevalence of autism and pervasive developmental disorders (PDD), from 14.7 to $30.8 / 10,000$ between 1980 and 1993, is reported in France. Morphogenetic anomalies (chromosomal, CNS and other anomalies), and hospitalization rates in the neonatal period are also increased in children with PDD (Guillem P et al. Dev Med Child Neurol November 2006;48:896-900). 